\title{
Membrane lipids in invadopodia and podosomes: Key structures for cancer invasion and metastasis
}

\author{
Hideki Yamaguchi ${ }^{1,2}$ and Tsukasa Oikawa ${ }^{3}$ \\ ${ }^{1}$ Growth Factor Division, National Cancer Center Research Institute, Tokyo 104-0045, Japan \\ 2 Precursory Research for Embryonic Science and Technology, Japan Science and Technology Agency, Saitama 332-0012, \\ Japan \\ ${ }^{3}$ Collaborative Research Resources, Institute for Integral Medical Research, School of Medicine, Keio University, Tokyo 160- \\ 8582, Japan \\ Correspondence to: Hideki Yamaguchi, e-mail: hidyamag@ncc.go.jp
}

Keywords: Invadopodia, podosome, cancer invasion, metastasis, lipid raft, phosphoinositide

Received: August 8, 2010, Accepted: September 4, 2010, Published: September 7, 2010

Copyright: (C) Yamaguchi et al. This is an open-access article distributed under the terms of the Creative Commons Attribution License, which permits unrestricted use, distribution, and reproduction in any medium, provided the original author and source are credited

\section{ABSTRACT:}

\begin{abstract}
Invadopodia are extracellular matrix (ECM)-degrading protrusions formed by invasive cancer cells. Podosomes are structures functionally similar to invadopodia that are found in oncogene-transformed fibroblasts and monocyte-derived cells, including macrophages and osteoclasts. These structures are thought to play important roles in the pericellular remodeling of ECM during cancer invasion and metastasis. Much effort has been directed toward identification of the molecular components and regulators of invadopodia/podosomes, which could be therapeutic targets in the treatment of malignant cancers. However, it remains largely unknown how these components are assembled into invadopodia/podosomes and how the assembly process is spatially and temporally regulated. This review will summarize recent progress on the molecular mechanisms of invadopodia/podosome formation, with strong emphasis on the roles of lipid rafts and phosphoinositides.
\end{abstract}

\section{INTRODUCTION}

Metastatic dissemination of cancer cells is the leading cause of mortality in patients with malignant cancers $[1,2]$. Cancer cells need to degrade the extracellular matrix $(\mathrm{ECM})$, which exists in the basement membrane, tumor stroma, and blood vessel walls, to emigrate from original tumor sites and invade adjacent tissues, and to eventually form metastatic sites at distant organs [3]. These processes seem to be facilitated by the formation of invadopodia, which are ventral membrane protrusions with ECM degradation activity formed by invasive cancer cells [4-8] (Fig. 1A and B). The ability of cancer cells to form invadopodia is closely related to their invasive and metastatic properties [9-11]. Additionally, during intravasation, invadopodia-like protrusions in cancer cells were observed in vivo by intravital imaging [12]. Furthermore, a recent study showed that invadopodia perforate the native basement membrane, allowing the invasive cancer cells to invade into the stroma [13]. Oncogene-transformed fibroblasts and cells of monocyte lineage also form functionally similar structures called podosomes that have ECM degradation activity (Fig. $1 \mathrm{C}$ and $\mathrm{D}$ ). The podosomes of macrophages/osteoclasts are used not only to elicit their physiological functions, but also to help cancer cells achieve efficient metastasis. Therefore, invadopodia/podosomes and their molecular regulators are considered as potential targets in the development of therapeutic strategies for cancer invasion and metastasis.

To date, many components of invadopodia have been reported, including proteins involved in the regulation of the actin cytoskeleton, cell signaling, cell-ECM adhesion, ECM degradation, and membrane remodeling [8, 14]. We and other researchers have previously proposed that invadopodia formation occurs in several steps $[9,13$, $15,16]$. Invadopodia precursors are assembled by actin polymerization machinery in response to extracellular stimuli. These structures are then stabilized by additional actin polymerization, and finally they gather matrix metalloproteinases to mature into functional invadopodia, which contain microtubules and intermediate filaments in addition to actin filaments. How these events occur at restricted sites on the plasma membrane of invasive 
cancer cells, however, is obscure. Recently, several studies regarding the role of membrane lipids in the regulation of invadopodia/podosome formation have been reported.

\section{LIPID RAFTS AND CAVEOLIN-1 IN INVADOPODIA FORMATION}

Lipid rafts are cholesterol- and sphingolipid-enriched membrane microdomains that are also referred to as lipid microdomains or detergent-resistant membranes (DRM). Lipid rafts have been implicated in a number of critical cellular processes, such as membrane transport and signal transduction [17, 18], as well as several pathological conditions, including cancer progression [19-21]. Caveolin-1 is a ubiquitously expressed scaffolding protein that is enriched in caveolae, which are subtypes of lipid rafts [22, 23]. Caveolin-1 is involved in several cellular functions such as endocytosis, vesicular transport, and signal transduction $[23,24]$.

Both we and Caldieri et al. recently reported that invadopodia are lipid raft-enriched domains in human
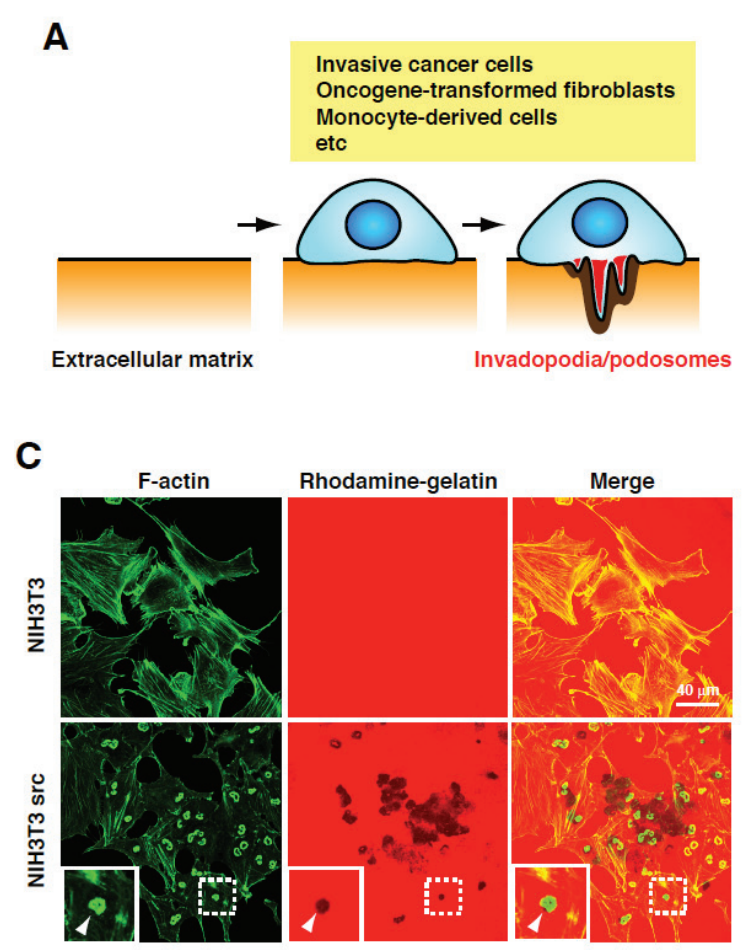

breast cancer and melanoma cells $[10,25]$. We also observed that lipid rafts were enriched at podosomes formed by Src-transformed fibroblasts (unpublished observations). The inhibition of lipid rafts by the depletion or sequestration of membrane cholesterol, or the blocking of glycosphingolipid synthesis, has been shown to impair invadopodia formation and function [10, 25]. Timelapse observation revealed that lipid raft membranes are actively trafficked and internalized around invadopodia, which indicates the possible involvement of lipid rafts in the transport of invadopodia components [10]. Several invadopodia components involved in actin polymerization and membrane trafficking, including neural WiskottAldrich syndrome protein (N-WASP), dynamin-2, and Arf6, are known to localize at lipid rafts [17, 26, 27]. Therefore, lipid rafts may act as platforms for localizing and activating these molecular machineries at the sites of invadopodia formation, which results in focalized ECM degradation.

The 2 studies also revealed that caveolin-1 is an essential regulator of the invadopodia-mediated

\section{B}

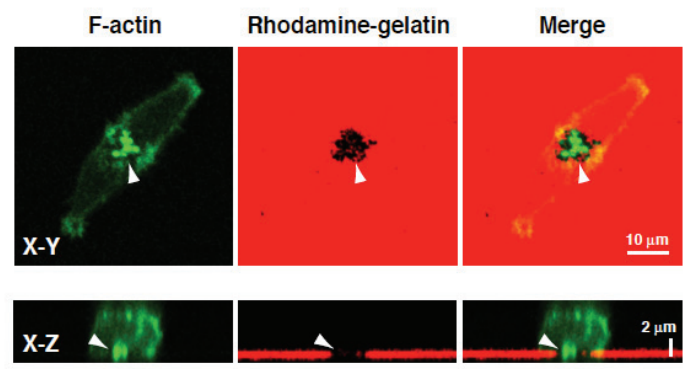

D

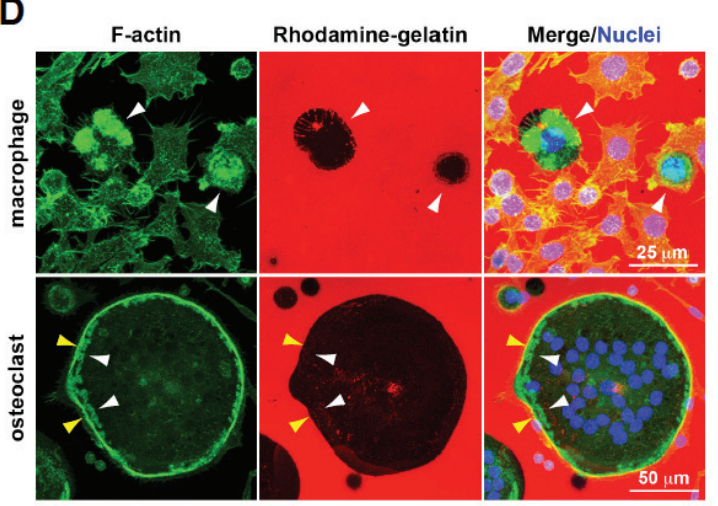

Figure 1: Invadopodia and podosomes formed by different cell types. (A) Schematic diagram of invadopodia/podosomes (B) Invadopodia formation by MDA-MB-231 human invasive breast cancer cells. The cells were cultured on rhodamine-gelatin-coated coverslips and stained with phalloidin to detect invadopodia that are enriched with actin filaments (F-actin). Upper and lower panels are confocal images showing XY and XZ sections, respectively. Invadopodia were observed as dot-like structures containing F-actin, which degrade the rhodaminegelatin matrix, resulting in the loss of gelatin fluorescence in the region of the invadopodia (arrowheads). (C) Podosomes formed by NIH3T3 cells transformed by constitutively active Src (NIH3T3 src). Parental NIH3T3 and NIH3T3 src cells were cultured and stained as described in (A). NIH3T3 src cells, but not parental NIH3T3 cells, form podosomes, which are observed as donut-like actin structures and colocalized with the gelatin degradation sites (arrowheads). (D) Podosome formation of macrophages and osteoclasts. RAW264.7 cells were cultured in the presence of lipopolysaccharide (LPS) $(100 \mathrm{ng} / \mathrm{ml})$ or RANKL $(10 \mathrm{ng} / \mathrm{ml})$ for $72 \mathrm{~h}$ to induce differentiation into the macrophages or osteoclasts, respectively. Cells were stained with phalloidin and 4',6-diamidino-2-phenylindole (DAPI). Macrophages form podosomes that often organize into large clusters associated with the gelatin degradation sites (arrowheads). Osteoclasts form a dense circumferential band of F-actin, called the sealing zone (yellow arrowheads), and clusters of podosomes that are observed inside the sealing zone (white arrowheads). A large gelatin degradation region was observed under these structures. 
degradation of ECM, which indicates that caveolin-1 plays an essential role in cancer cell invasion [10, 25]. Indeed, at least in breast cancer cell lines, caveolin-1 expression is predominantly observed in invasive cell lines and well correlated with invadopodia activity [10]. In melanoma cells, caveolin-1 functions at invadopodia through cholesterol transport to maintain proper levels of plasma membrane cholesterol [25]. Meanwhile, caveolin-1 is primarily involved in the transport of lipid raft-associated membrane type I matrix metalloproteinase (MT1-MMP), an invadopodia-enriched matrix metalloproteinase that is responsible for the ECM degradation activity of invadopodia [10]. Although further studies are needed to elucidate the precise functions of caveolin-1 in invadopodia formation, these findings imply that caveolin-1 plays multiple roles in the trafficking of invadopodia components. Clinical studies showed that the increased expression of caveolin-1 is correlated with the presence of metastasis and poor prognosis in several human cancers $[28,29]$. Taken together, blocking the functions of lipid rafts and caveolin-1 should be an approach to targeting invadopodia-mediated cancer cell invasion.

\section{PHOSPHOINOSITIDE SIGNALING INVADOPODIA FORMATION}

Phosphoinositides are membrane lipids that play multiple important roles in diverse cellular functions, such as membrane trafficking, signal transduction, cytoskeletal remodeling and deformation of the plasma membrane [30, 31]. Phosphoinositides are reversibly phosphorylated at the 3 different positions of the inositol headgroup. This generates 7 different species of phosphoinositide, namely, phosphatidylinositol 3-phosphate (PI3P), phosphatidylinositol 4-phosphate (PI4P), phosphatidylinositol 5-phosphate (PI5P), phosphatidylinositol 3,4-bisphosphate [PI(3,4)P2], phosphatidylinositol 4,5-bisphosphate [PI $(4,5) \mathrm{P} 2]$, phosphatidylinositol 3,5-bisphosphate $[\mathrm{PI}(3,5) \mathrm{P} 2]$, and phosphatidylinositol 3,4,5-trisphosphate [PI(3,4,5)P2]. There are a large number of enzymes that phosphorylate, dephosphorylate, or hydrolyze phosphoinositides to locally and temporarily regulate the levels of these phosphoinositide species [32-34]. As a result, each phosphoinositide has unique cellular distributions, which allows them to selectively recruit or activate target proteins that have specific phosphoinositide-binding domains [35]. $\mathrm{PI}(4,5) \mathrm{P} 2$ also acts as a precursor of second messengers: phospholipase C (PLC) hydrolyzes PI(4,5)P2 to generate diacylglycerol (DAG) and inositol 1,4,5-trisphosphate (IP3) and phosphoinositide 3-kinase (PI3-kinase) phosphorylates $\mathrm{PI}(4,5) \mathrm{P} 2$ to generate $\mathrm{PI}(3,4,5) \mathrm{P} 3[30$, 36]. Proteins that sever or depolymerize actin filaments in vitro, which include gelsolin, villin, cofilin, and profilin, are inactivated by $\mathrm{PI}(4,5) \mathrm{P} 2$ localized at the plasma membrane [37]. Conversely, proteins including vinculin, talin, ezrin/radixin/moesin (ERM) proteins, Wiskott-Aldrich syndrome protein (WASP)/N-WASP, and $\alpha$-actinin, which link actin filaments to each other or to the plasma membrane, are activated by this lipid $[37,38]$.

We recently reported that $\mathrm{PI}(4,5) \mathrm{P}_{2}$ is enriched at invadopodia and blockage of the $\mathrm{PI}(4,5) \mathrm{P}_{2}$ function suppresses invadopodia formation and ECM degradation by invasive human breast cancer cells [39]. We also found that a kinase generating $\mathrm{PI}(4,5) \mathrm{P}_{2}$, phosphatidylinositol4-phosphate 5-kinase type I $\alpha$ (PIPK I $\alpha$ ), accumulates at the invadopodia and that a knockdown of PIPK I $\alpha$ inhibits invadopodia formation. Importantly, the knockdown of PIPK I $\alpha$ only affects a pool of PI(4,5) $\mathrm{P}_{2}$, which is locally and newly produced by PIPK I $\alpha$. The knockdown of PIPK I $\alpha$ resulted in only a slight decrease in the total amount of $\mathrm{PI}(4,5) \mathrm{P}_{2}$, and did not affect the PI3-kinase signaling pathway, in which $\mathrm{PI}(4,5) \mathrm{P}_{2}$ acts as a major substrate. Therefore, $\mathrm{PI}(4,5) \mathrm{P}_{2}$ seems to exert its function via direct regulation of its own targets. Our previous study showed

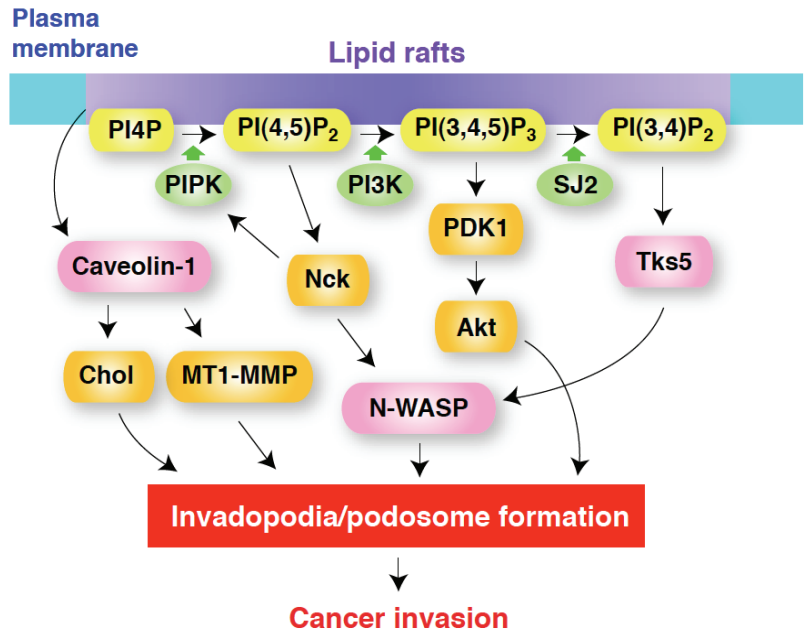

Figure 2: A model for the regulation of invadopodia/ podosome formation by membrane lipids. Lipid rafts may act as platforms for the recruitment of components of invadopodia/podosomes and for localized signaling by phosphoinositides. Caveolin-1 enriched in lipid rafts plays a role in cholesterol transport to maintain the levels of plasma membrane cholesterol (Chol) and also in MT1-MMP transport for the maturation of invadopodia. PI $(4,5) \mathrm{P}_{2}$ generated by PIP kinase type I $\alpha$ (PIPK) acts as a signaling molecule to locally activate several invadopodia components, including N-WASP, and also serves as a substrate of PI3-kinase (PI3K) for generation of $\mathrm{PI}(3,4,5) \mathrm{P}_{3}$, which in turn regulates invadopodia formation, most likely through PDK1 and Akt. Nck is required for N-WASP dependent actin polymerization induced by $\mathrm{PI}(4,5) \mathrm{P}_{2}$ and also stimulates PIPK for local enrichment of $\operatorname{PI}(4,5) \mathrm{P}_{2} . \operatorname{PI}(3,4) \mathrm{P}_{2}$, produced from $\mathrm{PI}(3,4,5) \mathrm{P}_{3}$ by the action of a specific phosphatase, possibly synaptojanin-2 (SJ-2), recruits Tks5/FISH to the plasma membrane, along with its binding partner N-WASP and other proteins involved in the formation of invadopodia/podosomes. It should be noted that the functions and requirements of these molecules may be slightly different between invadopodia and podosomes, as well as among cell types. 
that N-WASP and its activators, including Nck, are critical regulators of actin polymerization at the invadopodia core structures [9]. Because the activation of N-WASP is regulated by the amount of $\mathrm{PI}(4,5) \mathrm{P}_{2}$ on the plasma membrane [40], N-WASP is the most probable candidate for the $\mathrm{PI}(4,5) \mathrm{P}_{2}$ target. A recent study identified the existence of the reciprocal interdependence between Nck and $\mathrm{PI}(4,5) \mathrm{P}_{2}$ for regulation of N-WASP activity [41]. Nck is required for N-WASP-dependent actin polymerization induced by $\mathrm{PI}(4,5) \mathrm{P}_{2}$ and Nck also stimulates $\mathrm{PI}(4,5) \mathrm{P}_{2}$ production via recruitment of PIPK I $\alpha$. Considering that Nck and $\mathrm{PI}(4,5) \mathrm{P}_{2}$ are essential for invadopodia formation [9, 39], these components may interdependently activate N-WASP to assemble invadopodia structures. Other invadopodia components, such as cofilin and dynamin-2, are also regulated by $\mathrm{PI}(4,5) \mathrm{P}_{2}[31]$. Arf6 is known to activate PIPK $\mathrm{I} \alpha$ for $\mathrm{PI}(4,5) \mathrm{P}_{2}$ production [42]. Interestingly, synaptojanin-2, a $\mathrm{PI}(4,5) \mathrm{P}_{2} / \mathrm{PI}(3,4,5) \mathrm{P}_{3}$ phosphatase, is accumulated at invadopodia and required for invadopodia formation, which implies that this enzyme regulates the turnover of $\mathrm{PI}(4,5) \mathrm{P}_{2}$ at invadopodia [43]. Taken together, local $\mathrm{PI}(4,5) \mathrm{P}_{2}$ metabolism occurs at invadopodia and this seems to be a critical event to coordinate localization and activation of the invadopodia components. Classic biochemical studies have shown that phosphoinositide metabolism preferentially occurs within lipid raft fractions [44, 45]. Additionally, it has been shown that $\mathrm{PI}(4,5) \mathrm{P}_{2}$-dependent actin polymerization induced by N-WASP and Arp2/3 complex is initiated at the surface of the lipid raft-enriched membranes [46, 47]. Therefore, lipid rafts and phosphoinositides may cooperatively regulate invadopodia formation.

The PI3-kinases are a family of lipid kinases that phosphorylate phosphoinositides at the D-3 position of the inositol headgroup and thus produce D-3 phosphoinositides [48]. PI3-kinases mediate the signal transduction of extracellular stimuli and regulate diverse cellular events, such as mitogenesis, survival, membrane transport, and cell migration [36]. PI3-kinases are subdivided into 3 classes (I-III) in mammals on the basis of their enzyme domain structures and substrate specificity [33]. Uncontrolled activation of the PI3-kinase signaling pathway leads to several pathological phenomena, including tumorigenesis and tumor malignancies [36]. This is evidenced by the fact that the expression and activity of several members of the PI3-kinase signaling pathway are frequently altered in a variety of human cancers [49]. PI3-kinase activity is also required for invadopodia formation, as shown in invasive melanoma cells [50]. In line with this, we recently found that class IA PI3K catalytic subunit p110 $\alpha$ is selectively involved in invadopodia formation in breast cancer cells, and that PDK1 and Akt mediate the signaling (manuscript in submission). The PIK3CA gene, which encodes p110 $\alpha$, is one of the most frequently amplified and mutated genes identified in human cancers [49, 51]. Several clinical studies revealed that mutations leading to the activation of the PIK3CA gene are associated with invasive and metastatic phenotypes, as well as poor prognosis [5254]. Moreover, introduction of the mutant PIK3CA gene was reported to enhance the migration, invasion, and metastasis of breast cancer cells [55]. Therefore, p110 $\alpha$ is considered as a promising molecular target for the intervention of malignant cancers, and it has led to the development of several specific inhibitors [56].

\section{CELLS OF MONOCYTE ORIGIN GENERATE PODOSOMES, WHICH CONTRIBUTE TO CANCER CELL INVASION AND OSTEOLYTIC BONE METASTASIS}

Podosomes are F-actin-rich, dynamic adhesion structures found in Src-transformed cells (Fig. 1C) and the physiological context of monocyte-derived cells such as macrophages and osteoclasts (Fig. 1D). In the past decade, the generation of podosomes has been proven to be associated with the gene responsible for an $\mathrm{X}$ chromosome-linked immunodeficiency disease, WiskottAldrich syndrome (WAS). As macrophages from patients with WAS have defects in generating podosomes and polarization of the cell [57], these structures are thought to be important for chemotactic migration and/or the invasion of macrophages. Actually, the product of the gene (i.e., WASP) and its ectopic analogue, N-WASP, were shown to be indispensable for actin polymerization at podosomes via activating the Arp2/3 complex [57-61].

It is well established that the neoplastic properties of cancer cells are affected by interactions with the tumor microenvironment [62]. Tumor-associated macrophages (TAMs) have been implicated in tumor progression, metastasis, and poor prognosis in several human cancers [63,64]. A paracrine loop between macrophages and cancer cells has been proven to facilitate cancer cell migration and invasion both in vitro and in vivo, confirming the vicious role of TAMs $[65,66]$. Cancer cells stimulate the invasion of macrophages by secreting colony stimulating factor-1 (CSF-1), which in turn causes the macrophages to stimulate invasion of the cancer cells by secreting epidermal growth factor (EGF). EGF and CSF-1 are shown to stimulate invadopodia formation in cancer cells and podosome formation in macrophages, respectively $[6$, 9]. Therefore, the paracrine loop between cancer cells and TAMs may promote cancer progression $[63,64]$ partly via the formation of invadopodia/podosomes.

Osteoclasts are highly specialized multi-nucleated cells that are differentiated from the monocyte/ macrophage precursors on the bone surface in response to CSF-1 and receptor activator of nuclear-factor- $\mathrm{kB}$ ligand (RANKL). During the differentiation into mature cells, osteoclasts reorganize the actin cytoskeleton to form a dense circumferential band of F-actin (Fig. 1D). This 
ring forms a tight adhesive contact (the sealing zone) that defines a subcellular environment (which is known as a resorption pit or lacuna) into which $\mathrm{H}+$ and lytic enzymes are secreted, thereby allowing effective erosion of the bone $[58,67]$. The fully mature osteoclast can detach from the bone and move away from the resorption lacuna to participate in several rounds of resorption, which require podosome-associated cell motility $[67,68]$. One of the upstream regulators of WASP, the cytoplasmic kinase Src is essential for osteoclast activity in vivo, because Src knockout mice suffer from severe osteopetrosis caused by deficient osteoclast activity [69]. Osteoclasts derived from such mice cannot adhere and spread properly, and fail to give rise to mature sealing zones when attached to the bone. Bone metastases from breast cancer are typically osteolytic and cause destruction of the bone [70]. Breast cancer cells augment the activity of bone resorption via promoting the differentiation and podosome formation of osteoclasts by secreting transforming growth factor-beta (TGF- $\beta$ ), tumor necrosis factor-alpha (TNF- $\alpha$ ), interleukins (ILs), and parathyroid hormone-related protein (PTHrP), which leads to osteolytic bone metastasis.

\section{PRODUCTS OF PI3-KINASE REGULATE PODOSOME FORMATION}

$\mathrm{PI}(3,4,5) \mathrm{P}_{3}$, which is present in negligible amounts under resting conditions, is produced in the plasma membrane in response to extracellular stimuli; it is synthesized from $\mathrm{PI}(4,5) \mathrm{P}_{2}$ by the action of class I PI3kinase $[36]$. $\mathrm{PI}(3,4) \mathrm{P}_{2}$ is produced by the action of class I and II PI3-kinase on PI(4)P or via the dephosphorylation of $\mathrm{PI}(3,4,5) \mathrm{P}_{3}$ by $\mathrm{PI}(3,4,5) \mathrm{P}_{3}$ 5-phosphatases such as SHIP2 and synaptojanin-2 [31]. These locally produced $\mathrm{PI}(3,4,5)$ $\mathrm{P}_{3}$ and $\mathrm{PI}(3,4) \mathrm{P}_{2}$ recruit cytosolic proteins to the plasma membrane [35]. For example, the adhesion-mediated production of $\mathrm{PI}(3,4,5) \mathrm{P}_{3}$ stimulates protein complex formation, including PI3-kinase, Src, and gelsolin, which is mediated by direct interactions between $\mathrm{PI}(3,4,5) \mathrm{P}_{3}$ and the Src-homology 2 (SH2) domains of PI3-kinase and/ or Src [71]. Furthermore, phosphoinositides are able to modulate the functions of small GTPases of the Arf and Rho families, such as Rho, Rac, and Cdc42; these proteins are shown to be involved in podosome formation [7274]. This modulation occurs via the action of guanine nucleotide exchange factors (GEFs) or GTPase-activating proteins (GAPs) such as Tiam1, Vav1, and ASAP1 on these proteins; all these factors possess pleckstrin homology (PH) domains via which they interact with $\mathrm{PI}(4,5) \mathrm{P}_{2}$ and/ or $\mathrm{PI}(3,4,5) \mathrm{P}_{3}[75,76]$.

We investigated the localization of different species of phosphoinositides using various phosphoinositidebinding PH domains [77]. We demonstrated that $\mathrm{PI}(3,4)$ P2 is highly enriched in podosomes compared to the relatively diffused localization of $\mathrm{PI}(3,4,5) \mathrm{P} 3$, which is also found in lamellipodia and intracellular vesicles. What is intriguing is that excessive expression of the $\mathrm{PH}$ domain of Tapp1, which binds to $\mathrm{PI}(3,4) \mathrm{P} 2$, as well as the $\mathrm{PH}$ domain of Akt, which binds both to $\mathrm{PI}(3,4) \mathrm{P} 2$ and $\mathrm{PI}(3,4,5)$ P3, significantly suppressed podosome formation. This effect is thought to occur through sequestering those lipids by the domains, because the amount of protein expressed in a cell tends to correlate with the suppression effect. Furthermore, we found that $\mathrm{PI}(3,4) \mathrm{P} 2$ is synthesized by PI3-kinase and synaptojanin-2 in the vicinity of the focal adhesions, and that this phosphoinositide triggers the recruitment of a protein complex that includes Tks5, Grb2, and N-WASP, which results in the conversion of the adhesion sites to podosomes [77, 78]. Our results support the essential role of synaptojanin-2 in glioma cell migration and invasion [43], although the localization of $\mathrm{PI}(3,4) \mathrm{P} 2$ in glioma cells has not been determined. Tks5 is an adaptor protein with an N-terminal phox homology (PX) domain, which was originally identified as an Src substrate [79]. Both Tks5 and its relative, Tks4, have been shown to play important roles in podosome formation, matrix degradation, and tumor growth in vivo [80-83]. Recently, they have been shown to mediate the generation of reactive oxygen species (ROS) at the invadopodia of cancer cells, which is required for invadopodia formation and cancer cell invasion $[84,85]$. Moreover, Tks5 binds to supervillin, a lipid raft-enriched protein which is involved in integrin recycling, cell motility, and invadopodia formation [86-88], which suggests that they play roles as versatile regulators of invadopodia/podosomes. As the PX domain of Tks 5 binds to $\mathrm{PI}(3,4) \mathrm{P} 2$, and this interaction is essential for podosome formation downstream of Src [77, $82,83]$, targeting this interaction would be a promising therapeutic strategy for the selective intervention of cancer cell invasion and metastasis.

\section{CONCLUDING REMARKS}

As described above, accumulating evidence leaves us in no doubt that invadopodia/podosomes play a pivotal role in the invasion and metastasis of cancer cells. Moreover, podosomes formed by TAMs and osteoclasts in the tumor microenvironment seem to play supportive roles for cancer invasion and metastasis. The organization and components of the plasma membrane, such as lipid rafts and phosphoinositides, regulate the formation of invadopodia/podosomes. Therefore, targeting the molecular components of these structures, which include membrane lipids and their synthetic pathways, will contribute to the development of new strategies for the treatment of cancer invasion and metastasis.

One question that still remains answered is how lipid raft formation/degradation and phosphoinositide turnover are spatiotemporally regulated at invadopodia/podosomes. It is evident that invadopodia/podosomes are formed through several functional steps. Therefore, lipid rafts and phosphoinositide species may have distinct functions 
at different stages of invadopodia/podosome formation. Furthermore, although invadopodia and podosomes seem to share basic molecular components and functions, i.e., ECM degradation, their morphologies are quite different, even among cell types. If membrane lipids determine the site of invadopodia/podosome assembly, they may be critical determinants for the morphology, and most likely the function, of these structures. Further studies will be needed to address these questions.

\section{ACKNOWLEDGMENTS}

This work was supported by Grants-in-Aid for Scientific Research (B), Young Scientists (B), and the Promotion of Environmental Improvement for Independence of Young Researchers Project from the Ministry of Education, Culture, Sports, Science and Technology of Japan, and by a Grant-in-Aid from the Ministry of Health, Labour and Welfare of Japan for the $3^{\text {rd }}$ term Comprehensive 10-year Strategy for Cancer Control. This work was also supported in part by the Mochida Memorial Foundation for Medical and Pharmaceutical Research, the Uehara Memorial Foundation, Kao Foundation for Arts and Sciences, ONO Medical Research Foundation, and Takeda Science Foundation.

\section{REFERENCES}

1. Gupta GP, Massague J. Cancer metastasis: building a framework. Cell 2006; 127: 679-95.

2. Hanahan D, Weinberg RA. The hallmarks of cancer. Cell 2000; 100: 57-70.

3. KessenbrockK, Plaks V, WerbZ. Matrix metalloproteinases: regulators of the tumor microenvironment. Cell 2010; 141: 52-67.

4. Buccione R, Caldieri G, Ayala I. Invadopodia: specialized tumor cell structures for the focal degradation of the extracellular matrix. Cancer Metastasis Rev 2009; 28: 13749.

5. Linder S. The matrix corroded: podosomes and invadopodia in extracellular matrix degradation. Trends Cell Biol 2007; 17: 107-17.

6. Yamaguchi H, Pixley F, Condeelis J. Invadopodia and podosomes in tumor invasion. Eur J Cell Biol 2006; 85: 213-8.

7. Weaver AM. Invadopodia: specialized cell structures for cancer invasion. Clin Exp Metastasis 2006; 23: 97-105.

8. Gimona $\mathrm{M}$, Buccione $\mathrm{R}$, Courtneidge SA, Linder S. Assembly and biological role of podosomes and invadopodia. Curr Opin Cell Biol 2008; 20: 235-41.

9. Yamaguchi H, Lorenz M, Kempiak S, Sarmiento C, Coniglio S, Symons M, Segall J, Eddy R, Miki H, Takenawa $\mathrm{T}$, Condeelis J. Molecular mechanisms of invadopodium formation: the role of the N-WASP-Arp2/3 complex pathway and cofilin. J Cell Biol 2005; 168: 441-52.

10. Yamaguchi H, Takeo Y, Yoshida S, Kouchi Z, Nakamura Y, Fukami K. Lipid rafts and caveolin-1 are required for invadopodia formation and extracellular matrix degradation by human breast cancer cells. Cancer Res 2009; 69: 8594602.

11. Coopman PJ, Do MT, Thompson EW, Mueller SC. Phagocytosis of cross-linked gelatin matrix by human breast carcinoma cells correlates with their invasive capacity. Clin Cancer Res 1998; 4: 507-15.

12. Yamaguchi H, Wyckoff J, Condeelis J. Cell migration in tumors. Curr Opin Cell Biol 2005; 17: 559-64.

13. Schoumacher M, Goldman RD, Louvard D, Vignjevic DM. Actin, microtubules, and vimentin intermediate filaments cooperate for elongation of invadopodia. J Cell Biol 2010; 189: 541-56.

14. Yamaguchi H, Condeelis J. Regulation of the actin cytoskeleton in cancer cell migration and invasion. Biochim Biophys Acta 2007; 1773: 642-52.

15. Oser M, Yamaguchi H, Mader CC, Bravo-Cordero JJ, Arias M, Chen X, Desmarais V, van Rheenen J, Koleske AJ, Condeelis J. Cortactin regulates cofilin and N-WASp activities to control the stages of invadopodium assembly and maturation. J Cell Biol 2009; 186: 571-87.

16. Artym VV, Zhang Y, Seillier-Moiseiwitsch F, Yamada KM, Mueller SC. Dynamic interactions of cortactin and membrane type 1 matrix metalloproteinase at invadopodia: defining the stages of invadopodia formation and function. Cancer Res 2006; 66: 3034-43.

17. Hanzal-Bayer MF, Hancock JF. Lipid rafts and membrane traffic. FEBS Lett 2007; 581: 2098-104.

18. Simons K, Toomre D. Lipid rafts and signal transduction. Nat Rev Mol Cell Biol 2000; 1: 31-9.

19. Patra SK. Dissecting lipid raft facilitated cell signaling pathways in cancer. Biochim Biophys Acta 2008; 1785: 182-206.

20. Michel V, Bakovic M. Lipid rafts in health and disease. Biol Cell 2007; 99: 129-40.

21. Maxfield FR, Tabas I. Role of cholesterol and lipid organization in disease. Nature 2005; 438: 612-21.

22. Parton RG, Hanzal-Bayer M, Hancock JF. Biogenesis of caveolae: a structural model for caveolin-induced domain formation. J Cell Sci 2006; 119: 787-96.

23. Williams TM, Lisanti MP. The caveolin proteins. Genome Biol 2004; 5: 214.

24. Grande-Garcia A, del Pozo MA. Caveolin-1 in cell polarization and directional migration. Eur J Cell Biol 2008; 87: 641-7.

25. Caldieri G, Giacchetti G, Beznoussenko G, Attanasio F, Ayala I, Buccione R. Invadopodia biogenesis is regulated by caveolin-mediated modulation of membrane cholesterol levels. J Cell Mol Med 2009; 13: 1728-40.

26. Balasubramanian N, Scott DW, Castle JD, Casanova 
JE, Schwartz MA. Arf6 and microtubules in adhesiondependent trafficking of lipid rafts. Nat Cell Biol 2007; 9: 1381-91.

27. Otsuki M, Itoh T, Takenawa T. Neural Wiskott-Aldrich syndrome protein is recruited to rafts and associates with endophilin A in response to epidermal growth factor. J Biol Chem 2003; 278: 6461-9.

28. Quest AF, Gutierrez-Pajares JL, Torres VA. Caveolin-1: an ambiguous partner in cell signalling and cancer. J Cell Mol Med 2008; 12: 1130-50.

29. Burgermeister E, Liscovitch M, Rocken C, Schmid RM, Ebert MP. Caveats of caveolin-1 in cancer progression. Cancer Lett 2008; 268: 187-201.

30. Di Paolo G, De Camilli P. Phosphoinositides in cell regulation and membrane dynamics. Nature 2006; 443: 651-7.

31. Takenawa T, Itoh T. Phosphoinositides, key molecules for regulation of actin cytoskeletal organization and membrane traffic from the plasma membrane. Biochim Biophys Acta 2001; 1533: 190-206.

32. Anderson RA, Boronenkov IV, Doughman SD, Kunz J, Loijens JC. Phosphatidylinositol phosphate kinases, a multifaceted family of signaling enzymes. J Biol Chem 1999; 274: 9907-10.

33. Fruman DA, Meyers RE, Cantley LC. Phosphoinositide kinases. Annu Rev Biochem 1998; 67: 481-507.

34. Skwarek LC, Boulianne GL. Great expectations for PIP: phosphoinositides as regulators of signaling during development and disease. Dev Cell 2009; 16: 12-20.

35. Lemmon MA. Membrane recognition by phospholipidbinding domains. Nat Rev Mol Cell Biol 2008; 9: 99-111.

36. Cantley LC. The phosphoinositide 3-kinase pathway. Science 2002; 296: 1655-7.

37. Niggli V. Regulation of protein activities by phosphoinositide phosphates. Annu Rev Cell Dev Biol 2005; 21: 57-79.

38. Rohatgi R, Ma L, Miki H, Lopez M, Kirchhausen T, Takenawa T, Kirschner MW. The interaction between N-WASP and the Arp2/3 complex links Cdc42-dependent signals to actin assembly. Cell 1999; 97: 221-31.

39. Yamaguchi H, Yoshida S, Muroi E, Kawamura M, Kouchi Z, Nakamura Y, Sakai R, Fukami K. Phosphatidylinositol 4,5-bisphosphate and PIP5-kinase Ialpha are required for invadopodia formation in human breast cancer cells. Cancer Sci 2010; 101: 1632-8.

40. Papayannopoulos V, Co C, Prehoda KE, Snapper S, Taunton J, Lim WA. A polybasic motif allows N-WASP to act as a sensor of PIP(2) density. Mol Cell 2005; 17: 18191.

41. Rivera GM, Vasilescu D, Papayannopoulos V, Lim WA, Mayer BJ. A reciprocal interdependence between Nck and $\mathrm{PI}(4,5) \mathrm{P}(2)$ promotes localized N-WASp-mediated actin polymerization in living cells. Mol Cell 2009; 36: 525-35.

42. Funakoshi Y, Hasegawa H, Kanaho Y. Activation mechanisms of PIP5K isozymes by the small GTPase ARF6. Adv Enzyme Regul 2010; 50: 72-80.

43. Chuang YY, Tran NL, Rusk N, Nakada M, Berens ME, Symons M. Role of synaptojanin 2 in glioma cell migration and invasion. Cancer Res 2004; 64: 8271-5.

44. Pike LJ, Miller JM. Cholesterol depletion delocalizes phosphatidylinositol bisphosphate and inhibits hormonestimulated phosphatidylinositol turnover. J Biol Chem 1998; 273: 22298-304

45. Pike LJ, Casey L. Localization and turnover of phosphatidylinositol 4,5-bisphosphate in caveolin-enriched membrane domains. J Biol Chem 1996; 271: 26453-6.

46. Rozelle AL, Machesky LM, Yamamoto M, Driessens MH, Insall RH, Roth MG, Luby-Phelps K, Marriott G, Hall A, Yin HL. Phosphatidylinositol 4,5-bisphosphate induces actin-based movement of raft-enriched vesicles through WASP-Arp2/3. Curr Biol 2000; 10: 311-20.

47. Caroni P. New EMBO members' review: actin cytoskeleton regulation through modulation of $\mathrm{PI}(4,5) \mathrm{P}(2)$ rafts. EMBO J 2001; 20: 4332-6.

48. Engelman JA. Targeting PI3K signalling in cancer: opportunities, challenges and limitations. Nat Rev Cancer 2009; 9: 550-62.

49. Yuan TL, Cantley LC. PI3K pathway alterations in cancer: variations on a theme. Oncogene 2008; 27: 5497-510.

50. Nakahara H, Otani T, Sasaki T, Miura Y, Takai Y, Kogo M. Involvement of $\mathrm{Cdc} 42$ and Rac small $\mathrm{G}$ proteins in invadopodia formation of RPMI7951 cells. Genes Cells 2003; 8: 1019-27.

51. Zhao L, Vogt PK. Class I PI3K in oncogenic cellular transformation. Oncogene 2008; 27: 5486-96.

52. Li SY, Rong M, Grieu F, Iacopetta B. PIK3CA mutations in breast cancer are associated with poor outcome. Breast Cancer Res Treat 2006; 96: 91-5.

53. Maruyama N, Miyoshi Y, Taguchi T, Tamaki Y, Monden M, Noguchi S. Clinicopathologic analysis of breast cancers with PIK3CA mutations in Japanese women. Clin Cancer Res 2007; 13: 408-14.

54. Saal LH, Holm K, Maurer M, Memeo L, Su T, Wang X, Yu JS, Malmstrom PO, Mansukhani M, Enoksson J, Hibshoosh H, Borg A, Parsons R. PIK3CA mutations correlate with hormone receptors, node metastasis, and ERBB2, and are mutually exclusive with PTEN loss in human breast carcinoma. Cancer Res 2005; 65: 2554-9.

55. Pang H, Flinn R, Patsialou A, Wyckoff J, Roussos ET, Wu H, Pozzuto M, Goswami S, Condeelis JS, Bresnick AR, Segall JE, Backer JM. Differential enhancement of breast cancer cell motility and metastasis by helical and kinase domain mutations of class IA phosphoinositide 3-kinase. Cancer Res 2009; 69: 8868-76.

56. Knight ZA, Gonzalez B, Feldman ME, Zunder ER, Goldenberg DD, Williams O, Loewith R, Stokoe D, Balla A, Toth B, Balla T, Weiss WA, Williams RL, Shokat KM. A pharmacological map of the PI3-K family defines a role 
for p110alpha in insulin signaling. Cell 2006; 125: 733-47.

57. Linder S, Nelson D, Weiss M, Aepfelbacher M. WiskottAldrich syndrome protein regulates podosomes in primary human macrophages. Proc Natl Acad Sci USA 1999; 96: 9648-53.

58. Calle Y, Jones GE, Jagger C, Fuller K, Blundell MP, Chow J, Chambers T, Thrasher AJ. WASp deficiency in mice results in failure to form osteoclast sealing zones and defects in bone resorption. Blood 2004; 103: 3552-61.

59. Linder S, Higgs H, Hufner K, Schwarz K, Pannicke U, Aepfelbacher M. The polarization defect of WiskottAldrich syndrome macrophages is linked to dislocalization of the Arp2/3 complex. J Immunol 2000; 165: 221-5.

60. Mizutani K, Miki H, He H, Maruta H, Takenawa $\mathrm{T}$. Essential role of neural Wiskott-Aldrich syndrome protein in podosome formation and degradation of extracellular matrix in src-transformed fibroblasts. Cancer Res 2002; 62: 669-74.

61. de Noronha S, Hardy S, Sinclair J, Blundell MP, Strid J, Schulz O, Zwirner J, Jones GE, Katz DR, Kinnon C, Thrasher AJ. Impaired dendritic-cell homing in vivo in the absence of Wiskott-Aldrich syndrome protein. Blood 2005; 105: 1590-7.

62. Joyce JA, Pollard JW. Microenvironmental regulation of metastasis. Nat Rev Cancer 2009; 9: 239-52.

63. Condeelis J, Pollard JW. Macrophages: obligate partners for tumor cell migration, invasion, and metastasis. Cell 2006; 124: 263-6.

64. Qian BZ, Pollard JW. Macrophage diversity enhances tumor progression and metastasis. Cell 2010; 141: 39-51.

65. Goswami S, Sahai E, Wyckoff JB, Cammer M, Cox D, Pixley FJ, Stanley ER, Segall JE, Condeelis JS. Macrophages promote the invasion of breast carcinoma cells via a colony-stimulating factor-1/epidermal growth factor paracrine loop. Cancer Res 2005; 65: 5278-83.

66. Wyckoff J, Wang W, Lin EY, Wang Y, Pixley F, Stanley ER, Graf T, Pollard JW, Segall J, Condeelis J. A paracrine loop between tumor cells and macrophages is required for tumor cell migration in mammary tumors. Cancer Res 2004; 64: 7022-9.

67. Chellaiah MA, Soga N, Swanson S, McAllister S, Alvarez U, Wang D, Dowdy SF, Hruska KA. Rho-A is critical for osteoclast podosome organization, motility, and bone resorption. J Biol Chem 2000; 275: 11993-2002.

68. Chellaiah M, Kizer N, Silva M, Alvarez U, Kwiatkowski D, Hruska KA. Gelsolin deficiency blocks podosome assembly and produces increased bone mass and strength. J Cell Biol 2000; 148: 665-78.

69. Soriano P, Montgomery C, Geske R, Bradley A. Targeted disruption of the c-src proto-oncogene leads to osteopetrosis in mice. Cell 1991; 64: 693-702.

70. Akhtari M, Mansuri J, Newman KA, Guise TM, Seth P. Biology of breast cancer bone metastasis. Cancer Biol Ther 2008; 7: 3-9.
71. Chellaiah MA, Biswas RS, Yuen D, Alvarez UM, Hruska KA. Phosphatidylinositol 3,4,5-trisphosphate directs association of Src homology 2-containing signaling proteins with gelsolin. J Biol Chem 2001; 276: 47434-44.

72. Chellaiah MA. Regulation of actin ring formation by rho GTPases in osteoclasts. J Biol Chem 2005; 280: 32930-43.

73. Chellaiah MA. Regulation of podosomes by integrin alphavbeta3 and Rho GTPase-facilitated phosphoinositide signaling. Eur J Cell Biol 2006; 85: 311-7.

74. Santarius M, Lee CH, Anderson RA. Supervised membrane swimming: small G-protein lifeguards regulate PIPK signalling and monitor intracellular PtdIns(4,5)P2 pools. Biochem J 2006; 398: 1-13.

75. Rossman KL, Der CJ, Sondek J. GEF means go: turning on RHO GTPases with guanine nucleotide-exchange factors. Nat Rev Mol Cell Biol 2005; 6: 167-80.

76. D'Souza-Schorey C, Chavrier P. ARF proteins: roles in membrane traffic and beyond. Nat Rev Mol Cell Biol 2006; 7: 347-58.

77. Oikawa $\mathrm{T}$, Itoh $\mathrm{T}$, Takenawa $\mathrm{T}$. Sequential signals toward podosome formation in NIH-src cells. J Cell Biol 2008; 182: 157-69.

78. Oikawa T, Takenawa T. PtdIns(3,4)P2 instigates focal adhesions to generate podosomes. Cell Adh Migr 2009; 3: 195-7.

79. Lock P, Abram CL, Gibson T, Courtneidge SA. A new method for isolating tyrosine kinase substrates used to identify fish, an SH3 and PX domain-containing protein, and Src substrate. EMBO J 1998; 17: 4346-57.

80. Blouw B, Seals DF, Pass I, Diaz B, Courtneidge SA. A role for the podosome/invadopodia scaffold protein Tks5 in tumor growth in vivo. Eur J Cell Biol 2008; 87: 555-67.

81. Buschman MD, Bromann PA, Cejudo-Martin P, Wen F, Pass I, Courtneidge SA. The novel adaptor protein Tks4 (SH3PXD2B) is required for functional podosome formation. Mol Biol Cell 2009; 20: 1302-11.

82. Seals DF, Azucena EF, Jr., Pass I, Tesfay L, Gordon R, Woodrow M, Resau JH, Courtneidge SA. The adaptor protein Tks5/Fish is required for podosome formation and function, and for the protease-driven invasion of cancer cells. Cancer Cell 2005; 7: 155-65.

83. Abram CL, Seals DF, Pass I, Salinsky D, Maurer L, Roth TM, Courtneidge SA. The adaptor protein fish associates with members of the ADAMs family and localizes to podosomes of Src-transformed cells. J Biol Chem 2003; 278: 16844-51.

84. Diaz B, Shani G, Pass I, Anderson D, Quintavalle M, Courtneidge SA. Tks5-dependent, nox-mediated generation of reactive oxygen species is necessary for invadopodia formation. Sci Signal 2009; 2: ra53.

85. Gianni D, Diaz B, Taulet N, Fowler B, Courtneidge SA, Bokoch GM. Novel p47(phox)-related organizers regulate localized NADPH oxidase 1 (Nox1) activity. Sci Signal 2009; 2: ra54. 
86. Fang Z, Takizawa N, Wilson KA, Smith TC, Delprato A, Davidson MW, Lambright DG, Luna EJ. The membraneassociated protein, supervillin, accelerates F-actindependent rapid integrin recycling and cell motility. Traffic 2010; 11: 782-99.

87. Smith TC, Fang Z, Luna EJ. Novel interactors and a role for supervillin in early cytokinesis. Cytoskeleton (Hoboken) 2010; 67: 346-64.

88. Crowley JL, Smith TC, Fang Z, Takizawa N, Luna EJ. Supervillin reorganizes the actin cytoskeleton and increases invadopodial efficiency. Mol Biol Cell 2009; 20: 948-62. 